\title{
Switching "Real-World" Diabetes Patients to Degludec from Other Basal Insulins Provides Different Clinical Benefits According to Their Baseline Glycemic Control
}

\author{
Nikolaos Tentolouris · Søren T. Knudsen - Annunziata Lapolla • \\ Michael Lyng Wolden · Steffen Haldrup · Bernd Schultes
}

Received: December 14, 2018 / Published online: March 16, 2019

(C) The Author(s) 2019

\begin{abstract}
Introduction: The stable, ultra-long duration of action of insulin degludec (degludec) minimizes fluctuations in glucose-lowering activity over the daily (24-h) dosing period, and comparative studies with other basal insulins suggest that these properties translate into a lower risk of hypoglycemia at equivalent levels of glycemic control. Results from the real-world European multicenter, retrospective chart review study of
\end{abstract}

Enhanced Digital Features To view enhanced digital features for this article go to https://doi.org/10.6084/ m9.figshare.7718738.

Electronic supplementary material The online version of this article (https://doi.org/10.1007/s12325019-00916-7) contains supplementary material, which is available to authorized users.

N. Tentolouris $(\bowtie)$

National and Kapodistrian University of Athens, Athens, Greece

e-mail: ntentolouris@yahoo.gr

S. T. Knudsen

Aarhus University Hospital, Aarhus, Denmark

A. Lapolla

University of Padova, Padua, Italy

M. L. Wolden · S. Haldrup

Novo Nordisk A/S, Søborg, Copenhagen, Denmark

B. Schultes

eSwiss Medical and Surgical Center, St Gallen, Switzerland
2550 patients with type 1 and type 2 diabetes (T1D and T2D) in routine clinical care EUTREAT (NCT02662114) showed that patients benefited from improved glycemic control and significantly reduced rates of hypoglycemia following a switch to degludec.

Methods: In this post hoc analysis, EU-TREAT patients were stratified into good $(\leq 7.5 \%$ HbA1c), intermediate $(>7.5$ to $\leq 8.5 \% \mathrm{HbA} 1 \mathrm{c})$, and poor ( $>8.5 \% \mathrm{HbA1c}$ ) glycemic control at baseline to investigate the possibility of differential benefits, either improved control or reduced risk of hypoglycemia, whichever the need. Changes in HbA1c, overall hypoglycemia, and total insulin dose from baseline to 6 and 12 months follow-up were assessed for each group.

Results: For both T1D and T2D patients, those in good initial control experienced significant reductions in rates of hypoglycemia and total insulin dose following the switch, without compromising control. Those in poor initial control achieved significant improvements in HbA1c with no change in rates of hypoglycemia or total insulin dose.

Conclusion: This analysis expands the findings of EU-TREAT by showing differential changes in the clinical endpoints depending on particular need. It introduces the possibility that the differential benefits of degludec could address two of the renowned clinical challenges faced when treating diabetes: improving glycemic control for optimal management of T1D and titrating 
insulin dose in T2D, both without fear of increased hypoglycemia.

Trial Registration: ClinicalTrials.gov, NCT02 662114.

Funding: Novo Nordisk A/S.

Keywords: EU-TREAT study; Glycemic control; Hypoglycemia; Insulin degludec; Long-acting insulin; Observational study; Post hoc analysis; Type 1 diabetes; Type 2 diabetes

\section{INTRODUCTION}

Insulin degludec (degludec) is a basal insulin with a unique mode of protraction, conferring an ultra-long duration of action (exceeding $42 \mathrm{~h}$ at relevant clinical doses in patients with type 1 diabetes, T1D) and a stable glucose-lowering profile over the 24-h dosing period [1-3]. Effective insulin therapy commonly requires dose intensification, yet this process is often restricted by experience and/or fear of hypoglycemic events [4, 5], their accompanying negative physical, societal, and psychological consequences, and loss of productivity [4-8]. In a multinational, cross-sectional survey of physician and patient attitudes to insulin, the majority (88\%) of responding physicians admitted many patients fell short of target HbA1c levels, yet dose increases were hindered because of fear of hypoglycemia [5, 7]. This exemplifies the "barrier of hypoglycemia" [4] which can compromise treatment [9].

The European multicenter, retrospective, non-interventional chart review study EUTREAT (NCT02662114) was a real-world study of 2550 patients with diabetes (1717 with T1D and 833 with type 2 diabetes, T2D) in routine clinical care across six countries in Europe. EUTREAT investigated the changes in clinical characteristics (HbA1c, hypoglycemia, and total insulin dose) that followed a switch to degludec from another basal insulin [10]. As EU-TREAT was a large-scale study of the use of degludec in routine clinical practice, the data encompass the impact of factors such as clinical setting, provider, lifestyle, environment, and health system on the outcomes of treatment with degludec. Thus, the data can address different questions to the standard efficacy and safety investigations of randomized clinical trials $[11,12]$. Mean results showed that in both T1D and T2D, switching was associated with improved glycemic control, reduced rates of hypoglycemia, and reduced total insulin doses [10]. As around one-third of patients were already in good control at baseline (36.9\% T1D and $28.5 \%$ T2D had HbA1c $<7.5 \%$ ), the switch to degludec was not driven solely by a desire to improve HbA1c [10]. Switching was motivated by blood glucose variability in approximately $70 \%$ of patients and it is possible that the preswitch insulin prompted a heterogeneous response, whereby some patients achieved good glycemic control at the cost of suffering hypoglycemic events, while others achieved tolerability at the cost of suboptimal control [10]. Most modern studies of insulin treatments have a treat-to-target design, investigating differences in hypoglycemic events at HbA1c parity. Predominantly compared with insulin glargine U100, degludec has consistently been shown to reduce hypoglycemic risk in such studies $[10,13-16]$. It seems logical that patients with control restricted by the "barrier of hypoglycemia" [4] could safely switch to degludec and improve control, whereas those in good control yet experiencing hypoglycemia could switch to improve tolerability whilst maintaining control.

In this post hoc analysis we have used data from EU-TREAT to test if the complementary benefits (improved glycemic control or reduced rates of hypoglycemia, dependent on individual need) are achieved in a real-world setting. As baseline HbA1c has been identified as the strongest predictor of achieving glycemic control, we performed the analysis on data from EU-TREAT patients stratified into three groups of different levels of baseline HbA1c [17].

\section{METHODS}

The detailed study design and methodology of the EU-TREAT study have been described previously [10]. To summarize, data were collected from the medical records of patients with T1D or T2D across Europe who had been treated 
with degludec after switching from another basal insulin [10].

Patients were required to have switched to degludec $[ \pm$ oral antidiabetic drugs (OADs) \pm prandial insulin] from any other basal insulin ( \pm OADs \pm prandial insulin) at least 6 months before data collection [10]. Here, we stratified patients into the following categories of glycemic control at baseline, chosen to represent an equal number of patients and reflect common real-world clinical scenarios, where patients are not necessarily treated to HbA1c $<7 \%$ :

- Good control (HbA1c $\leq 7.5 \%)$

- Intermediate control (HbA1c $>7.5$ to $\leq 8.5 \%)$

- Poor control (HbA1c > 8.5\%)

Endpoints were change in HbA1c, overall hypoglycemia, and total insulin dose from baseline to 6 and 12 months' follow-up. A hypoglycemic event was defined as any episode of hypoglycemia recorded by the healthcare providers in patient charts [10]. The EU-TREAT study was conducted in accordance with the Declaration of Helsinki (2013 amendment) and written informed consent from all patients was obtained before enrolment. The study protocols were approved according to local regulations by appropriate health authorities and by institutional review boards (see Supplementary Table S1) at all participating institutions. The analysis reported here does not contain any studies with human participants or animals performed by any of the authors.

\section{Statistical Analysis}

Analyses of baseline characteristics, demographics, and primary data have been described previously [10]. Data were recorded to reflect two periods of medical history: before (preswitch) and after (post-switch) the date of degludec initiation. Baseline was defined as the most recent recording during the 3-month period prior to switch.

Pre- and post-switch outcome data were collected at $6 \pm 3$ and $12 \pm 3$ months prior to and following switch, as per methodology described previously [10]. Six-month data were available for all patients with T1D and T2D; 12 -month data were available for $76 \%$ of those with T1D and $72 \%$ of those with T2D [10]. Changes in HbA1c and mean daily insulin doses at the +6 -month and +12 -month time points were analyzed using analysis of covariance (ANCOVA), and the - 12-month and - 6month data were used to validate results. The changes were modeled as a function of the baseline value and relevant covariates that included country, age, body mass index (BMI), gender, diabetes duration, duration of insulin therapy, and type of basal injections. Leastsquares (LS) mean estimates for the changes were reported, with associated 95\% confidence intervals and $p$ values as appropriate [10]. The number of hypoglycemic events per patientmonth at baseline was estimated from counts of events during the 6 months pre-switch. Numbers of events per patient-month at the +6 month and +12 -month time points were estimated from counts during these periods [10]. Rate data were analyzed using a negative binomial regression estimator, which included a variable to capture differential exposure across patients and a variable to capture the effects of pre- and post-switch [10].

\section{RESULTS}

\section{Baseline and Clinical Characteristics}

A total of 2550 patients were included in the study (T1D, $n=1717$; T2D, $n=833$ ). Baseline demographics and clinical characteristics of stratified patient groups are presented in Table 1.

\section{Subgroup Clinical Endpoints}

The 6- and 12-month changes in HbA1c, hypoglycemia rates, and insulin doses for the stratified T1D and T2D patient groups are shown in Fig. 1i, ii, respectively. At both time points, T1D and T2D patients in good glycemic control at baseline benefited from reductions in rates of hypoglycemia and total insulin dose while still maintaining glycemic control. For 


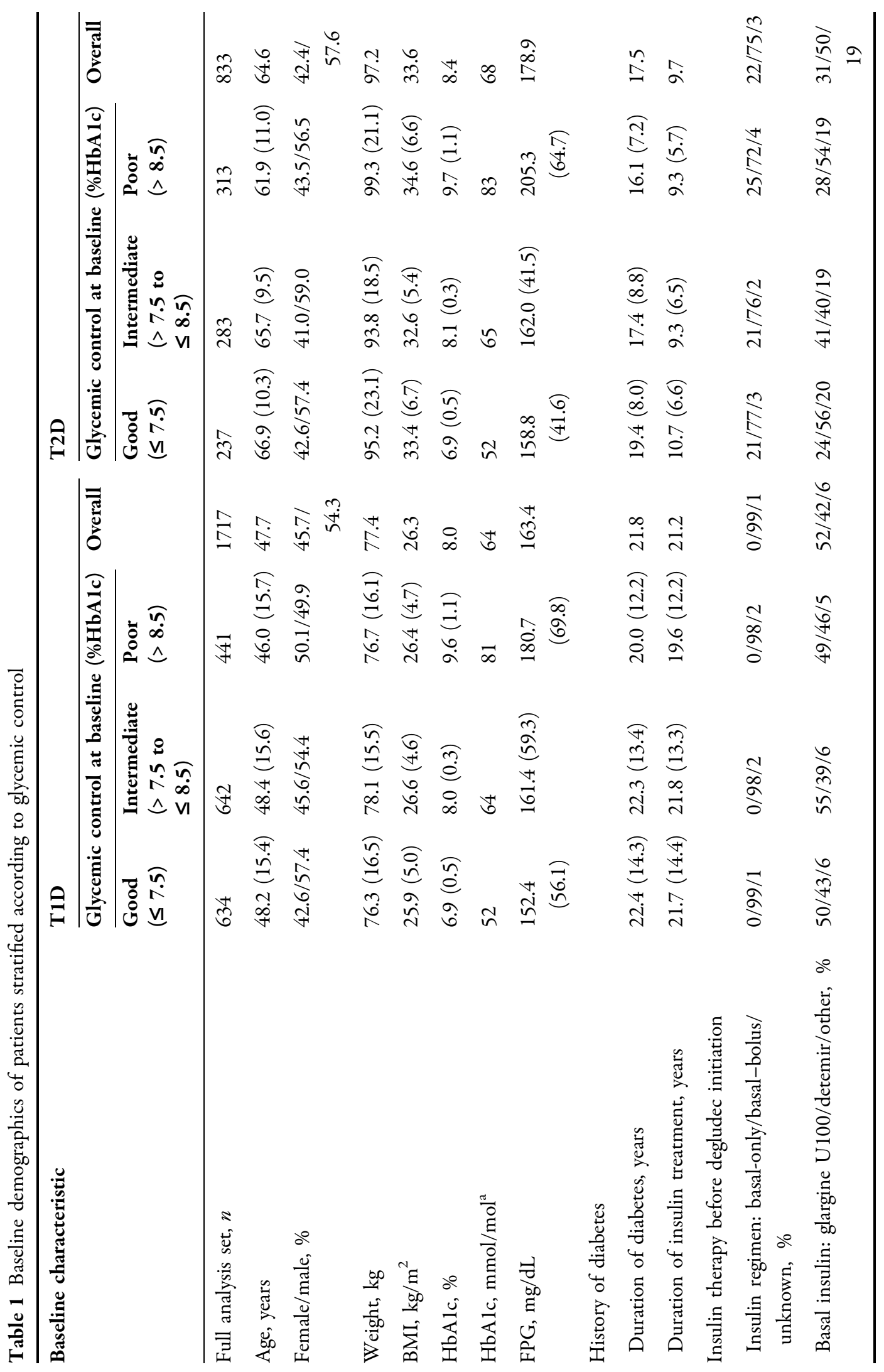




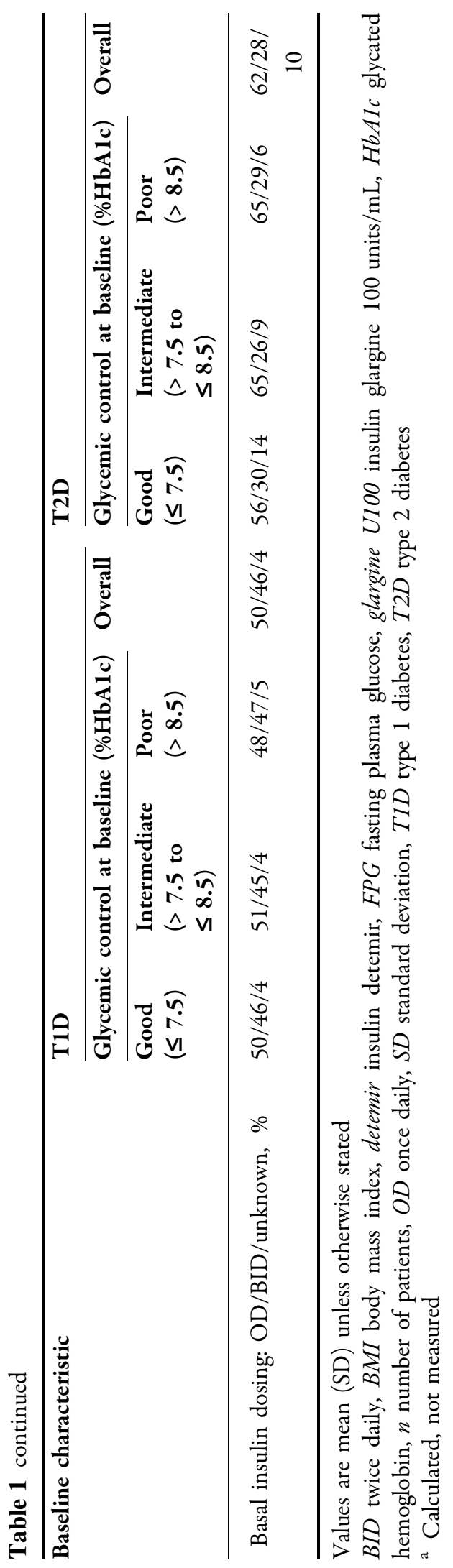

those with T1D ( $n=634$; mean HbA1c 6.9\%), there was a minor, clinically insignificant mean increase in $\mathrm{HbA1c}(0.1 \%)$, and reductions in rates of hypoglycemia were $16 \%$ at 6 months and $23 \%$ at 12 months. For those with T2D in good control $(n=237)$, large rate reductions of $67 \%$ and 73\%, respectively, were achieved $(p<0.05)$. Total insulin dose for T1D and T2D patients in good control was reduced by 5.4 and 6.5 mean units, respectively, at 6 months, with similar results at 12 months $(p<0.05)$.

T1D and T2D patients in intermediate glycemic control at baseline benefited from both reductions in mean $\mathrm{HbA1c}(-0.2 \%$ and $-0.3 \%$, respectively) and in rates of hypoglycemia (27\% and $66 \%$, respectively) at 6 months, with similar results at 12 months $(p<0.05)$.

All patients in poor glycemic control at baseline benefited from clinically significant (in excess of $-0.64 \%$ ) mean reductions in HbA1c, with reductions in excess of $-1.05 \%$ for patients with T2D, at both 6 and 12 months $(p<0.05)$.

For all stratified patients with T1D, insulin dose and rates of hypoglycemia were significantly reduced by at least $10 \%$ at both 6 and 12 months, although the reduction in hypoglycemia at 12 months was relatively small $(10 \%)$ and not statistically significant for those in poor control ( $p=0.5583$ ) (Fig. 1i).

Concerning concomitant OADs, there were no changes in mean dosage or number of agents used, and the number of prandial insulin injections for T2D patients recorded at the point of switching to degludec and thereafter remained comparable.

\section{DISCUSSION}

This post hoc analysis expands the findings from EU-TREAT by showing how changes in clinical endpoints varied between patients of different glycemic status at baseline. We have discerned that patients most in need of further glycemic control achieved improvements greater than the mean, while patients most in need of reduced hypoglycemic risk also achieved benefits beyond the mean. Each group 


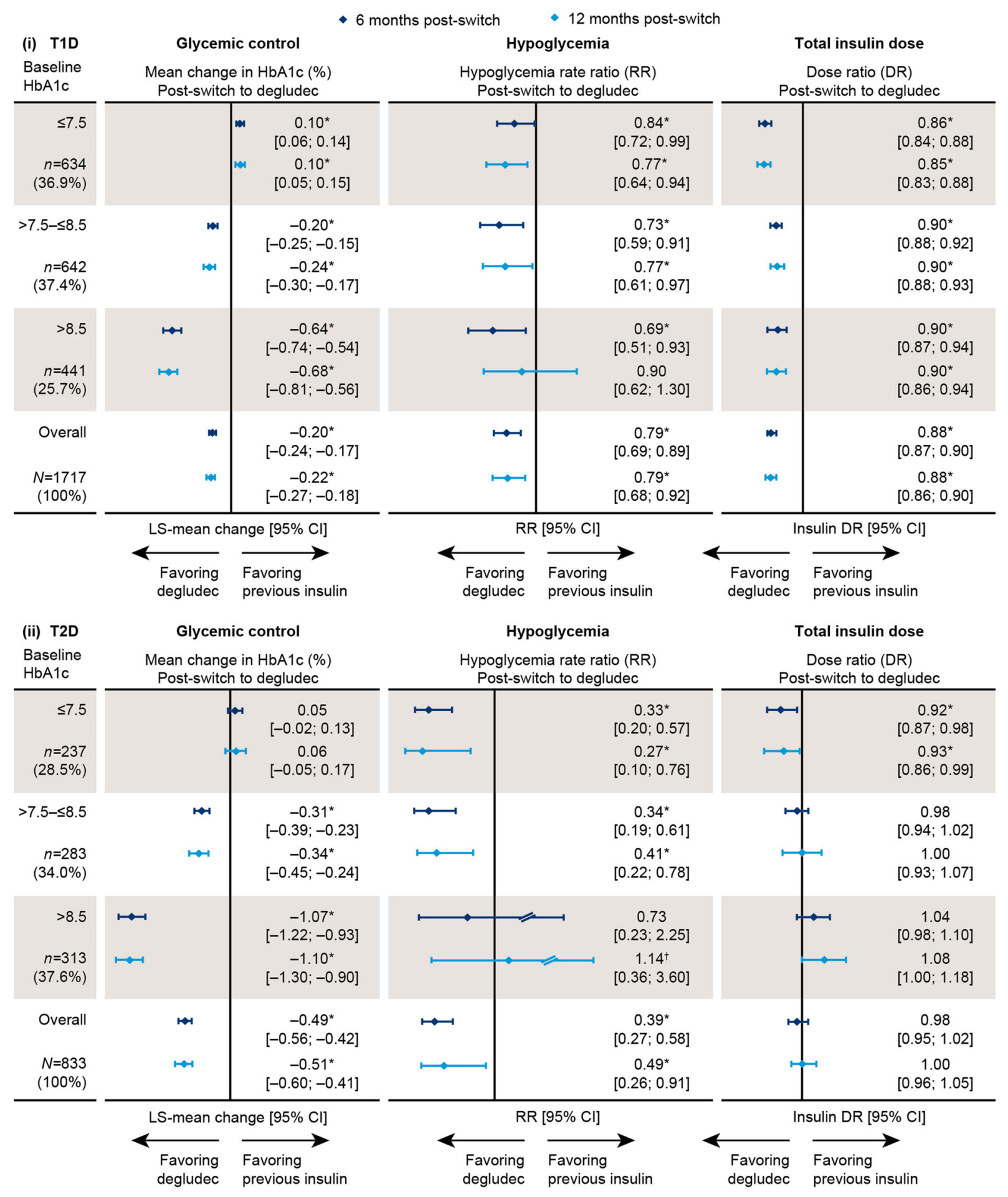


4Fig. 1 Changes in clinical outcomes at 6 and 12 months postswitch for patients with (i) T1D and (ii) T2D in the EUTREAT trial stratified according to baseline glycemic control. $\leq 7.5=$ good glycemic control; $>7.5-\leq 8.5=$ intermediate glycemic control; $>8.5=$ poor glycemic control. ${ }^{*} p<0.05$, †estimated using Poisson regression due to convergence issues. All changes are from baseline. $n=$ number of patients within each $\mathrm{HbAlc}$ group, $n=$ total number of T1D/T2D patients. Analysis of glycemic control: data are LSmean change. Multivariate ANCOVA model controlled for country (when appropriate), age, BMI, gender, diabetes duration, duration of insulin therapy, and type of basal injections. Analysis of hypoglycemia: negative binomial regression model controlled for age, BMI, gender, diabetes duration, and duration of insulin therapy. Analysis of total insulin dose: data are log-transformed dose ratios (6- or 12-month total insulin dose/baseline total insulin dose) based on units per kilogram of body weight. Multivariate ANCOVA model controlled for country (when appropriate), age, BMI, gender, diabetes duration, duration of insulin therapy, and type of basal injections. ANCOVA analysis of covariance, BMI body mass index, CI confidence interval, DR dose ratio, LS least-squares, $\mathrm{RR}$ rate ratio, T1D type 1 diabetes, T2D type 2 diabetes

better fulfilled their respective clinical needs, without compromising the other endpoint.

Hypoglycemia is a problematic treatment complication. The global Hypoglycemia Assessment Tool (HAT) observational study of more than 27,000 insulin-treated patients with diabetes revealed incidences more than 10-fold higher than previously reported, of 73.3 (T1D) and 19.3 (T2D) events per patient-year [9].

Hypoglycemia is a life-long risk in T1D, which requires full insulin replacement therapy, and the average patient suffers several asymptomatic and symptomatic episodes each week [4]. These episodes are commonly triggered by bolus insulin, which was taken by all T1D patients accounted for in this study and could have influenced the smaller rate reduction experienced by those in good control, compared with T2D patients $(16 \%$ vs. $67 \%$ at 6 months). The reduction is impressive nonetheless, given T1D patients' increased hypoglycemic vulnerability, and our results are consistent with observations in the SWITCH 1 and SWITCH 2 studies [14, 15].

Currently, the only proven clinical option for improving T1D control without introducing additional hypoglycemic risk is insulin pump therapy, but results from this post hoc analysis present switching to degludec as a promising alternative. Although a very minor $(0.1 \%)$ but statistically significant increase in HbA1c was seen among patients with T1D and in good glycemic control, this is below the level of $0.5 \%$ which physicians are reported to consider a clinically relevant change [18]. This group also had the highest percentage reductions in insulin dose and significant reductions in hypoglycemic risk, which could reflect a conservative clinical strategy: physicians may have reduced treatment intensity to contain further risk of hypoglycemia.

In late-stage T2D following initiation of insulin treatment, the risk, frequency, and severity of hypoglycemic episodes tend to increase substantially, to approach the T1D scenario [4]. The barrier of hypoglycemia responsible for delaying insulin initiation and reluctance to intensify dose can result in protracted hyperglycemia and attendant morbidity [4]. The significant reduction in rates of hypoglycemia experienced by T2D patients in good control following a switch to degludec introduces an opportunity to circumvent this barrier with a treatment that achieves glycemic control without an increase or even a reduction in the risk of hypoglycemia [4, 9]. Patients with T2D in poor initial glycemic control benefited from the greatest improvements across all stratified groups. This could partly be due to the fear of dose intensification resulting in their prior maintenance on lower-than-optimal doses of pre-switch insulin. It might be anticipated that this fear may diminish following switch to degludec, as patients experience improvements in control without additional hypoglycemic events [5]. Most of these patients were at an advanced stage of disease, with $70 \%$ on bolus therapy, and therefore difficult to treat because of increased hypoglycemic risk [4]. As such, they were likely to have had the greatest scope for improvement and therapy intensification. In T2D patients overall, the benefits observed can confidently be ascribed to degludec, as there were no changes in concomitant OAD regimens or in the use of other insulins.

Limitations of this analysis mirror those of the primary study, including its observational, 
retrospective design and the lack of a comparator arm, which prevented investigating how the effects observed after switching to degludec would compare to switching to an alternative basal insulin. Collection of data was restricted to those data recorded in patient medical charts, which probably included only a subset of the true number of hypoglycemic events. Such under-recording of hypoglycemic events, however, would likely have been equal across the three stratified groups and therefore not impact the calculated rate ratios. The restricted data, together with limitations in analysis, have made it impossible to capture the periodic fluctuations in blood glucose levels that prompted the switch to degludec for most patients despite their adequate average HbA1c control. Despite these limitations, our data nevertheless represent a valuable addition to findings from the degludec clinical trials [13].

There are two noteworthy advantages: as the primary study was a real-world study conducted outside the closely controlled clinical trial setting, its findings have high external validity. There is growing interest in the potential of realworld evidence to complement findings from randomized clinical trials, as the latter are not generalizable to the diverse group of patients found in normal clinical practice $[11,12]$. In addition, this was a large-scale study of a significant number of patients. The few other published observational studies involving a switch to degludec are limited in size, population (T1D or T2D), and duration of follow-up [19-23]. One smaller-scale study, however, has findings that support our results. Of 211 patients with T2D switched from alternative basal insulins to degludec, those in poor control $(\mathrm{HbA} 1 \mathrm{c}>8.5 \%)$ benefited from a greater reduction in HbA1c compared with the overall population $(1.0 \pm 1.1 \%$ vs. $0.58 \pm 1.0 \%$, $p<0.001)$ [19].

\section{CONCLUSION}

In routine clinical practice, switching from other basal insulins to degludec offers differential clinical benefits for patients with diabetes, according to baseline glycemic control. Patients who switch while in good control benefit from significant reductions in rates of hypoglycemia and total insulin dose, without compromising control. Patients who switch while in poor glycemic control achieve significant improvements in control, with no increase in rates of hypoglycemia or total insulin dose.

\section{ACKNOWLEDGEMENTS}

We thank the participants of the EU-TREAT study (NCT02662114) on which this article is based. The authors thank Charlotte Thim Hansen MD and Deniz Tutkunkardas MD (Novo Nordisk) for their review and input to the manuscript.

Funding. These analyses were funded by Novo Nordisk A/S, along with the open access and article-processing charges for this paper. EU-TREAT (NCT02662114) was funded by Novo Nordisk A/S. All authors had full access to all of the data in this study and take complete responsibility for the integrity of the data and accuracy of the data analysis.

Medical Writing and Editorial Assistance. Medical writing assistance and editorial/submission support were provided by Elizabeth Hilsley and Richard McDonald of Watermeadow Medical, an Ashfield Company, part of UDG Healthcare plc, funded by Novo Nordisk A/S.

Authorship. All named authors meet the International Committee of Medical Journal Editors (ICMJE) criteria for authorship for this article, take responsibility for the integrity of the work as a whole, and have given their approval for this version to be published.

Prior Presentation. Some of the data in this manuscript have been presented in poster form at the 100th Annual Meeting of the Endocrine Society (ENDO 2018), 17-20 March 2018, Chicago, IL, USA.

Disclosures. Nikolaos Tentolouris has participated in advisory panels for AstraZeneca, 
Boehringer Ingelheim, Eli Lilly, ELPEN, Merck Sharp Dohme (MSD), Novartis, Novo Nordisk and Sanofi, and has received research support from MSD, Eli Lilly, Novo Nordisk A/S, Sanofi, Pfizer, AstraZeneca, Janssen, Cilag, GlaxoSmithKline and Novartis. Søren T. Knudsen has participated in advisory panels for, and has received honoraria for lectures from Novo Nordisk, Amgen, Eli Lilly, Sanofi, MSD, Boehringer Ingelheim and AstraZeneca. Michael Lyng Wolden is an employee of and shareholder in Novo Nordisk. Steffen Haldrup is an employee of and shareholder in Novo Nordisk. Bernd Schultes has participated in advisory panels for AstraZeneca, Sanofi, Novo Nordisk, Eli Lilly and Boehringer Ingelheim, and has received honoraria for lectures from MSD, AstraZeneca, Sanofi, Novo Nordisk, Eli Lilly and Boehringer Ingelheim. Annunziata Lapolla has nothing to disclose.

Compliance with Ethics Guidelines. The EU-TREAT study was conducted in accordance with the Declaration of Helsinki (2013 amendment) and written informed consent from all patients was obtained before enrolment. The study protocols were approved according to local regulations by appropriate health authorities and by institutional review boards (see Supplementary Table S1) at all participating institutions. The analysis reported here does not contain any studies with human participants or animals performed by any of the authors.

Data Availability. The datasets generated during and/or analyzed during the current study are available from the corresponding author on reasonable request.

Open Access. This article is distributed under the terms of the Creative Commons Attribution-NonCommercial 4.0 International License (http://creativecommons.org/licenses/ by-nc/4.0/), which permits any noncommercial use, distribution, and reproduction in any medium, provided you give appropriate credit to the original author(s) and the source, provide a link to the Creative Commons license, and indicate if changes were made.

\section{REFERENCES}

1. Heise T, Hermanski L, Nosek L, Feldman A, Rasmussen S, Haahr $\mathrm{H}$. Insulin degludec: four times lower pharmacodynamic variability than insulin glargine under steady-state conditions in type 1 diabetes. Diabetes Obes Metab. 2012;14(9):859-64.

2. Jonassen I, Havelund S, Hoeg-Jensen T, Steensgaard DB, Wahlund PO, Ribel U. Design of the novel protraction mechanism of insulin degludec, an ultra-long-acting basal insulin. Pharm Res. 2012;29(8):2104-14.

3. Heise T, Nosek L, Bottcher SG, Hastrup H, Haahr H. Ultra-long-acting insulin degludec has a flat and stable glucose-lowering effect in type 2 diabetes. Diabetes Obes Metab. 2012;14(10):944-50.

4. Cryer PE. The barrier of hypoglycemia in diabetes. Diabetes. 2008;57(12):3169-76.

5. Frier BM, Jensen MM, Chubb BD. Hypoglycaemia in adults with insulin-treated diabetes in the UK: selfreported frequency and effects. Diabet Med. 2016;33(8):1125-32.

6. Brod M, Christensen T, Thomsen TL, Bushnell DM. The impact of non-severe hypoglycemic events on work productivity and diabetes management. Value Health. 2011;14(5):665-71.

7. Peyrot M, Barnett AH, Meneghini LF, SchummDraeger PM. Insulin adherence behaviours and barriers in the multinational Global Attitudes of Patients and Physicians in Insulin Therapy study. Diabet Med. 2012;29(5):682-9.

8. Pawaskar M, Iglay K, Witt EA, Engel SS, Rajpathak S. Impact of the severity of hypoglycemia on healthrelated quality of life, productivity, resource use, and costs among US patients with type 2 diabetes. J Diabetes Compl. 2018;32(5):451-7.

9. Khunti K, Alsifri S, Aronson R, et al. Rates and predictors of hypoglycaemia in 27585 people from 24 countries with insulin-treated type 1 and type 2 diabetes: the global HAT study. Diabetes Obes Metab. 2016;18(9):907-15.

10. Siegmund T, Tentolouris N, Knudsen ST, et al. A European, multicentre, retrospective, non-interventional study (EU-TREAT) of the effectiveness of insulin degludec after switching basal insulin in a population with type 1 or type 2 diabetes. Diabetes Obes Metab. 2018;20:689-97.

11. Sherman RE, Anderson SA, Dal Pan GJ, et al. Realworld evidence-what is it and what can it tell us? N Engl J Med. 2016;375(23):2293-7. 
12. Bate A, Juniper J, Lawton AM, Thwaites RM. Designing and incorporating a real world data approach to international drug development and use: what the UK offers. Drug Discov Today. 2016;21(3):400-5.

13. Ratner RE, Gough SC, Mathieu C, et al. Hypoglycaemia risk with insulin degludec compared with insulin glargine in type 2 and type 1 diabetes: a preplanned meta-analysis of phase 3 trials. Diabetes Obes Metab. 2013;15(2):175-84.

14. Lane W, Bailey TS, Gerety G, et al. Effect of insulin degludec vs insulin glargine U100 on hypoglycemia in patients with type 1 diabetes: the SWITCH 1 randomized clinical trial. JAMA. 2017;318(1):33-44.

15. Wysham C, Bhargava A, Chaykin L, et al. Effect of insulin degludec vs insulin glargine u100 on hypoglycemia in patients with type 2 diabetes: the SWITCH 2 randomized clinical trial. JAMA. 2017;318(1):45-56.

16. Marso SP, McGuire DK, Zinman B, et al. Efficacy and safety of degludec versus glargine in type 2 diabetes. N Engl J Med. 2017;377(8):723-32.

17. Riddle MC, Vlajnic A, Zhou R, Rosenstock J. Baseline HbA1c predicts attainment of $7.0 \% \mathrm{HbA} 1 \mathrm{c}$ target with structured titration of insulin glargine in type 2 diabetes: a patient-level analysis of 12 studies. Diabetes Obes Metab. 2013;15(9):819-25.

18. Lenters-Westra E, Schindhelm RK, Bilo HJ, Groenier $\mathrm{KH}$, Slingerland RJ. Differences in interpretation of haemoglobin A1c values among diabetes care professionals. Neth J Med. 2014;72(9):462-6.

19. Melzer Cohen C, Thorsted BL, Wolden ML, Chodick G, Karasik A. Improved glycemic control achieved by switching to insulin degludec in insulin-treated patients with type 2 diabetes in a realworld setting: a non-interventional, retrospective cohort study. Diabetes Ther. 2017;8(5):1047-55.

20. Hamamoto Y, Honjo S, Fujimoto K, et al. Basal and bolus insulin dose changes after switching basal insulin to insulin degludec in patients with type 1 diabetes mellitus: a pilot study. Clin Drug Investig. 2017;37(9):845-52.

21. Takahashi N, Tsujimoto T, Inoue K, Kishimoto M, Kajio H. Improvement of glycemic control without severe hypoglycemia in a type 1 diabetes patient undergoing hemodialysis after a change from insulin glargine to insulin degludec. J Diabetes Investig. 2016;7(5):805-6.

22. Evans M, McEwan P, Foos V. Insulin degludec early clinical experience: does the promise from the clinical trials translate into clinical practice-a casebased evaluation. J Med Econ. 2015;18(2):96-105.

23. Landstedt-Hallin L. Changes in HbA1c, insulin dose and incidence of hypoglycemia in patients with type 1 diabetes after switching to insulin degludec in an outpatient setting: an observational study. Curr Med Res Opin. 2015;31(8):1487-93. 